\title{
A Comparative Evaluation Study of Basic Interaction Techniques for PDAs in Point-Of-Care Situations
}

\author{
Ole Andreas Alsos \\ Dept. of Computer and Information Science \\ Norwegian University of Science and Technology \\ Trondheim, Norway \\ ole.andreas.alsos@idi.ntnu.no
}

\author{
Benjamin Dabelow \\ Medical Faculty \\ University of Heidelberg \\ Heidelberg, Germany \\ benjamin@dabelow.eu
}

\begin{abstract}
This qualitative usability evaluation study has compared interaction techniques of a paper based medical chart and three versions of a PDA based system, based on interaction with stylus, finger or hardware buttons. Users' preferences and opinions were collected from 56 simulated hospital ward rounds with 14 physicians. Despite a number of disadvantages compared to paper the users preferred PDAbased interaction techniques. Within the PDA-based interaction techniques the physicians' preferences showed large variations. Moreover, the techniques had different qualities in different situations. The study identifies $\mathbf{1 4}$ factors influencing the users' preference and shows how each interaction techniques accommodate each factor. This study can inform the design and choice of interaction techniques on new handheld point-of-care systems.
\end{abstract}

Keywords; Interaction techniques, mobile computing, user interfaces, usability, point of care, comparative study, experimental simulation, usability evaluation.

\section{INTRODUCTION}

Physicians and nurses have for several years used small handheld devices, such as PDAs, as a replacement for penand-paper and medical reference books [1]. Such devices have in recent years received wide adoption in health care and are increasingly used for ubiquitous clinical information access [2]. However, to further enhance user acceptance [3], and to reduce medical errors in the process of entering and retrieving information [4], hardware and software design must be improved. While much work has been done to improve and optimize clinical software designs for handhelds, few studies have reported which interaction technique is optimal for use in a point-of-care setting.

Physicians, who are the primary users of such systems in hospitals, often have a strong standing in their institution, and have a large degree of freedom in their choice of work methods and organization $[5,6]$. They often choose what tools they want to use, and they also have the power not to use tools that do not accommodate their needs. They can leave the handheld device in the desktop drawer, use oldfashioned pen-and-paper, or rely on their excellent memory if they want.

Given the independency of physicians in their selection of tools, the interaction design of mobile clinical information

Permission to make digital or hard copies of all or part of this work for personal or classroom use is granted without fee provided that copies are not made or distributed for profit or commercial advantage and that copies bear this notice and the full citation on the first page. To copy otherwise, to republish, to post on servers or to redistribute to lists, requires prior specific permission and/or a fee. PERVASIVEHEALTH 2010, March 22-25, Munchen, Germany

Copyright $\odot 2010$ ICST 978-963-9799-89-9

DOI 10.4108/ICST.PERVASIVEHEALTH2010.8835 system is of particularly high importance to assure user acceptance and success of the implementation.

While established guidelines and principles exist for the specification of GUIs [7, 8], few such guidelines exist when it comes to choosing and specifying the interaction techniques used by the system. Such decisions are mainly based on quantitative measures [9]. However, in healthcare there are other factors, social and physical ones, which are equally important for the overall usability of a mobile information system [10]. Take a ward round as an example. Here, factors such as the ability of the doctor to attend the patient rather than the user interface [11], the ability of the patient to be aware of the doctor's actions [12], and the ability of the doctor to communicate non-verbally with the device [13], are important to allow "sensitive or full patient disclosure" [14]. Whether one interaction technique is $10 \%$ faster than another is of less importance. Therefore, we have in this study mainly focused on qualitative measures, such as the doctors' and patients' preferences and concerns about the interaction techniques.

Our main research goal with this study has been to find out which interaction technique a PDA-based point-of-care medication system should employ. To find the answer, we have evaluated commonly used basic interaction techniques and compared them with their current paper based counterpart in a series of ward round simulations with physicians and patient actors.

\section{A. Background}

While most usability evaluation studies have covered a single system, several examples of comparative usability evaluations of multiple design alternatives are found in literature. Traditionally, when performing comparative studies of interaction techniques, time completion rate, error rate and other quantitative measurements are used to decide which design to move forward with. One of the earliest attempts in the HCI-field was conducted during the development of the Star user interface [9] back in the 1980's, where several design concepts were developed using comparative evaluations. A standard for something as trivial as selecting, copying and pasting text using a mouse was not yet established, so the Star team designed several different solutions for text selection and exposed them to users. The 
team measured the completion times of each solution, refined the "winner", and used it in new design iterations.

The usability design lifecycle [8] represents a similar process: Explore the design space by developing several different design solutions, evaluate them, and use the best designs or design elements as a basis for a new or improved solution.

Similar studies have been performed within the health care domain. One study made a direct comparison of Tablet PCs and PDAs for point-of-care documentation using focus groups and usability walk-troughs with end-users [15]. They found that the amount and complexity of the data to be entered were important factors for the physicians' preference. Tablet computers were better suited for complex high volume data entry, while PDAs were preferred for simple low volume data entry.

Another study found that some users used their finger or the hardware buttons to operate handheld prototypes when comparing interaction techniques for a PDA-based health information system, even when the system under test was designed for stylus usage [16]. This demonstrates that the users do not necessarily consider the stylus as the optimal solution for point-of-care usage.

A review of studies comparing paper and PDA demonstrated that handhelds in general are a faster and preferred alternative to paper-based data collection [17]. However, another study found that paper-based media has much richer interaction capabilities compared to electronic media, such as PCs and PDAs [18].

\section{CANDIDATE INTERACTION TECHNIQUES}

In this study we have compared the three most common techniques for interacting with a small handheld computer. The techniques are supported by most current PDA models. As a baseline we present the current paper-based practice (Fig. 1.).

\section{A. Stylus.}

The stylus is a small and thin pen-like device used to point at and interact with GUI elements displayed on the PDA touch screen. It is designed to fit into a slot in the handheld device for storage when not in use.

Very few studies on PDA-based medical applications explicitly report which interaction style was used. However, judging from screenshots and the size of the user interface controls of a selection of PDA-based health information

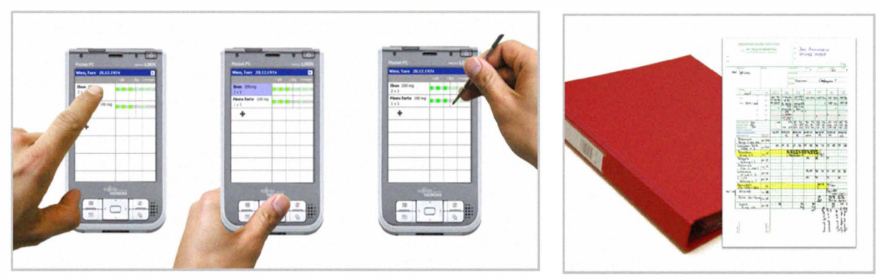

Figure 1. The three candidate interaction techniques finger, buttons, stylus and the paper chart. systems (reported by [3]) they all use the stylus as the main interaction technique.

\section{B. Finger.}

Handheld devices with touch screens also allow the users to user their fingertip or nail to interact with the device, although with reduced accuracy.

A number of mobile phone vendors have recently started producing smart-phones with touch screens that are particularly designed for finger based interaction (e.g. iPhone) proving the practicability of this approach. This has resulted in a wide range of simple personal health applications based on finger interaction for touch based smart phones. We have not found any other studies describing PDA-based systems specifically designed for finger usage in the health care domain. However, there are several examples from other domains (e.g. [19]).

\section{Buttons.}

Most PDAs also allow interaction with the system through hardware buttons, which are positioned below or around the touch screen.

Recently smart-phone vendors have produced devices that enable the users to slide out a full hardware qwerty-keyboard in addition to the touch screen. Some health care systems (presented in [3]) exist in versions for mobile phones, using only hardware buttons for text entry and navigation.

\section{Current paper based practice.}

The general concept of a paper-based medical-chart has been developed over centuries of medical practice. In the large regional university hospital in Norway where this study was conducted, "The Chart" (as it is widely named by its users) is a collection of important medical documents about the patient, gathered in a binder. It is used as documentation when the health worker is visiting the patient [20]. The main document is an A4-sized form containing the most important information about the patient, such as blood pressure, body temperature, prescribed medication, etc. In addition the binder contains other documents such as recent test results or reports.

\section{METHODS}

When performing formative evaluations of mobile systems it can be beneficial to do so in realistic environments. If doing otherwise, one might find usability problems in the graphical user interface, but will fail to find critical usability problems concerning the physical and social aspects of usability $[10,21]$. In ward round situations field studies are often considered the optimal research method, but due to issues such as patient safety, time cost, and the lack of control they are not always the method of choice [21, 22].

\section{A. Experimental design.}

For this experiment we utilized a usability laboratory where we recreated the hospital environment and evaluated the interaction techniques in a "hospital ward simulator" with 
real physicians who played out realistic ward round scenarios. The general setup was a simulated ward round where a physician made changes in the medication of patient impersonators using four different information systems; three PDA-based medication systems and one paper based medical chart.

\section{B. Location and recording equipment.}

The tests were conducted in a usability laboratory for testing medical systems at a research center connected to the regional hospital area. The laboratory is a full-scale model of a section of a hospital ward with two patient rooms, one office for health workers and one hallway connecting the rooms. Only one of the patient rooms was used in the experiment. Adjacent to the lab area is a control room with recording and editing equipment.

The PDA's screen content was wirelessly mirrored and mixed real-time together with video streams from four cameras and audio streams from three wireless microphones. Three of the cameras were mounted in the ceiling and were remotely controllable from the control room. The fourth camera, a non-intrusive mini camera, was mounted on the bedside, giving a video stream from the patient's perspective. All together the recorded video provided details about the overall care situation and the physician's interaction with the GUI, with the physical device, and with the patient. Fig. 2 shows the resulting video stream.

\section{Handheld information system.}

PDAs are better for low volume and simple data entry [15]. Therefore, the prototypes were deliberately designed with only four functions; prescribe a new medication, change, pause, or cease treatment of a prescribed medication. The first function required more effort (select medication, medication dose and medication administration) than the three other (select prescribed medication and change it). In the medication selection process we simulated an idealized form of context dependency to reduce the length of the medication list. The list only presented medications that were reasonable to be prescribed in the particular scenario. Each task required only 3 or 4 buttons to be pressed in the user interface.

The user interface was designed using minimal attention user interface principles [19], and the symbols and icons used were already well known from the paper chart or other applications (screenshots are found in Fig. 3, left and middle). In the simulations, we used a Fujitsu Siemens Power Loox. This PDA offers input with stylus, finger, and hardware buttons.

Three versions of the prototype were developed, each adapted for interaction with stylus, finger and device buttons. The differences between them were minor; the finger prototype had larger GUI-buttons than the stylus prototype, and the button prototype had an indicator moving between GUI-buttons as the user navigated through them with the hardware buttons (the touch screen was disabled).

The main rationale for making the handheld medication prescription system basic and simple with few functions and few screens was that the number of navigation and usability problems often increases with number of functions and navigational complexity.

\section{Paper based interaction technique.}

The paper based interaction technique used in the simulations employed the paper chart as in use today (Fig. 3, right). Domain experts, a senior physician and a nurse, were involved to create a realistic paper chart for the fictive patients used in the scenarios. However, it contained only the same information that was available on the PDA.

\section{E. Test order.}

A within-subject testing approach was chosen to limit the number of tests and test subjects. Thus each participant tested all user interfaces. The order of the tests was rotated between the tests to control and reduce possible order and learning effects. To reduce the number of patient impersonators, the two patient cases were alternated so that patient scenario A and patient impersonator $\mathrm{A}$ was used with the first and third interaction method while patient scenario $\mathrm{B}$ and patient impersonator B was used with the second and fourth. Pilot testing with physicians not part of the study revealed that they were able to play the scenario realistically despite repeating the scenarios.

\section{F. Test participants.}

Fourteen physicians participated in the study. They were all recruited from a large regional hospital and were paid for their participation. Their age ranged from 25 to $60(M=41,4$ / $\mathrm{SD}=11,7)$, with an even distribution of male and female.

Three sociology students on a graduate level (used because of their ability to observe and report social behavior and interaction) and a last year $\mathrm{PhD}$ student in health informatics acted as patients during the tests. The patient actors were aged from 26 to $47(\mathrm{M}=33,0 / \mathrm{SD}=9,9)$. Three of

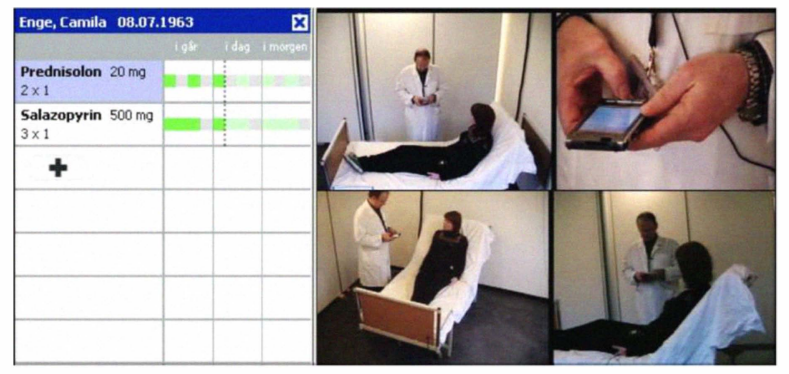

Figure 2. Frame from video data showing a physician's interaction with the GUI (left), with the physical device (upper right), and with the patient and the overall care situation (middle and lower right).

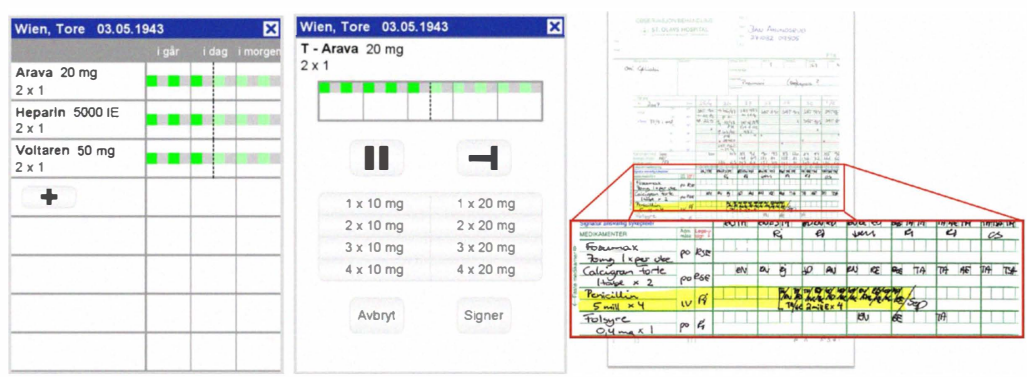

Figure 3. Screenshots from the stylus prototype showing the main screen (left), the change/pause/cease screen (middle) and the paper chart used in the experiments (right). 
them were female and one was male.

\section{G. Patient scenario objectives.}

Two patient scenarios were developed in cooperation with domain experts (a physician and nurse). The purpose of the patient scenarios was (1) to provide a realistic clinical situation, (2) to employ the physicians' professional experience and practices, (3) to reduce the scope of the dialog in the consultation, (4) to reduce variations in the outcome and (5) to make sure the physicians had at least one interaction with the PDA or paper chart during the visit, triggered by the patients' complaints and concerns. One of the scenarios is provided below:

The patient is a 44-year-old woman hospitalized with an acute episode of Crohn's disease since two days. The treatment was started right after admission of the patient. It comprises (1) Prednisolon (Prednisolone), $20 \mathrm{mg}$, tablet, twice a day, and (2) Salazopyrin (Sulfasalazine), $500 \mathrm{mg}$, tablet, three times a day. During the ward round the patient discloses to the physician that she has developed an itching rash over her whole body. She remembers that she had a similar reaction to some antibiotic, but does not remember its name.

\section{H. Instructions to physicians.}

As part of the preliminary briefing we explained that the motivation for the experiment was to "test a user interface for a medication module for an electronic patient record system". Each participant was also given a guided presentation of the laboratory and the associated control room. They were also presented with the general tasks to carry out during the tests. Since the purpose was not to find usability errors of the system as such, they were able to familiarize with the prototypes prior to the experiment.

In the preliminary briefing all the test subjects were encouraged to communicate and interact with the patient impersonators during the test, as they would have done with actual patients in real clinical situations.

\section{Instructions for patient impersonators.}

Before the arrival of the physician, the patient impersonators were given detailed instructions how to behave according to the patient scenarios and asked to memorize this. They were not given any explanation of the research questions being studied.

\section{J. Test execution.}

At the beginning of each test, the participant impersonator was lying in the bed in the patient room. In the hallway the physician was reminded of the case description of the next patient case and the user interface she would be using. This recapitulation was repeated for each new case, while the next patient impersonator was assuming his position in the patient bed.

\section{K. Physician post-test interview.}

After completing all tests, the physician was interviewed about his or her experiences during the test and about their opinions regarding mobile computing in hospitals in general. The interview was performed in a semi-structured form with some predefined questions, but the physician was encouraged to raise other issues of personal concern in the context of the test.

The different prototypes were discussed in the order they were used in the test. To facilitate discussion and to avoid misunderstandings, cards with symbolic pictures of the different prototypes were provided for reference. They also had the paper chart and PDA available.

The second part of the interview focused more on general and open questions about the physician's opinion on potential distraction by the information devices, suitability for real life usage and the patients' anticipated perception of PDAs at the point-of-care.

After debriefing the participants, they were asked to fill in a questionnaire on personal data, job details, and their previous experience with computer and PDA usage. Afterwards the physicians were given the opportunity for an informal concluding chat.

\section{Card ranking}

During the interview the physicians were asked to rank the interaction techniques devices in a card sort exercise, where they ordered the cards representing the interaction techniques by preference (ties between cards were not allowed). They were asked to state the reason for their final rank order.

\section{Patient focus group.}

When all 14 physicians had tested the four interaction techniques, we arranged a focus group where the patient actors participated. During this session we asked them about their observations and experiences as patients.

\section{N. Analysis}

\section{1) Video data from usability evaluation}

Given that usability problems of mobile devices often go beyond the graphical user interface [10], we made detailed observations of (1) usability problems related to the user interfaces, (2) usability issues regarding the different interaction techniques, (3) issues regarding the physical and bodily aspects of usability, and (4) issues regarding the social aspects of usability. The video data from the experiments were transcribed and analyzed by two observers.

\section{2) Physician interviews and patient focus group}

The interviews were transcribed and analyzed to find the physicians' opinions and concerns about each technique. The interviews data were analyzed in software for qualitative studies (QSR NVIVO 8) using an approach inspired by grounded theory [23].

\section{3) Preference Ranking}


The card ranking data was used to calculate a quantitative measure of the physicians' interaction technique preferences. The preference ranking of each user was coded from 1 (least preferred) to 4 (most preferred). A Friedman test was used to reveal if there were any significant rank differences in the data set, and a Mann-Whitney test was applied to each pair of interaction technique to reveal any significant rank differences between them.

\section{RESULTS}

The ward round simulations offered a realistic view on how the physicians used the paper and PDA at the point-ofcare. Together with data from the survey, card ranking exercise, patient focus group, and physician interviews, we were able to get a good impression of the qualities of the different interaction techniques. First we present general results from the survey, preference ranking and patient focus group. Next, detailed findings from the observation and physician interviews are presented.

\section{A. Survey}

The survey revealed that the participants' level of experience with computers was very good. They all used computers daily, both in their job and privately. One participant reported using computers privately weekly only.

The level of experience with handheld computers, such as PDAs, was generally very low. Only 2 of the participants used PDAs or other handheld computers daily in their job, while the rest used it rarely or never. Two physicians used a handheld computer daily in private, one weekly and the rest rarely or never.

\section{B. Preference ranking}

Table I shows the preference ranking results. The total score illustrate that stylus and finger were preferred over buttons and paper. Paper was the least preferred interaction technique. The Friedman test uncovered significant rank differences in the data set $(p=0,009, \mathrm{CF}=95 \%)$. However, the Mann-Whitney test revealed only significant rank differences between paper and stylus/finger (see Table II).

The card ranking provoked the users reflection by reminding them what they had just used. Instead of having to remember the differences, this knowledge was provided to them through the cards. We also observed that very few referred to the interaction techniques by name - they rather referred to it by pointing at the card or the device, which was available during the card sort.
When sorting the cards in the preferred order, the physicians reasoned and argued for their decision. This revealed what the important usability factors were for them. These factors, and the way the interaction technique accommodated them, decided the position of the card in the stack. The aspects mentioned during the card sort are reported together with the interview results in section $\mathrm{D}$.

\section{Patient focus group}

The patient focus group revealed that the patient actors did not notice any differences between the various PDA based medication systems. However, they claimed to experience "more embarrassing silence" during the simulated ward rounds when the physicians used the PDA. This silence prevented them from asking questions because they did not want to disturb the physician. They found it easier to ask questions when using the paper chart.

The patients also perceived physicians as being more confident and comfortable when using the paper chart, something that made them trust the physicians more. This was visible in the observation data through more eye contact and a livelier conversation while writing in the chart. On the other hand, the patient experienced the PDA as less invasive because it took less space between the patient and physician.

However, due to the low number of patients and the fact that they were not real patients, these results should be used as nothing else than an indication of patient experience and a direction for further work on how patients perceive handhelds compared to paper charts.

\section{Ward round simulation and physician interviews}

In general, the physicians found the mobile device input faster and its content easier to read compared to handwriting. Some found the devices almost as simple as paper. One of them said; "This was quite simple to use. If I tried it for weeks or months it would be as simple as the one I'm used to [the chart]." However, most of them were worried about software malfunctions, usability problems or other situations forcing them to spend a lot of time fiddling with the device.

Because they already had access to medication names and dosages, the chances of making mistakes were reduced. In addition, mistakes were easier to correct. One of the physicians said; "From a quality perspective I would prefer the PDA, because I tend to misspell things. That makes me look up things, but [the PDA] does this automatically."

Several liked the physical attributes of the handheld device; it was easy to carry and it fit in their pockets. On the other hand, the small screen gave a limited view of
TABLE I. TEST SUBJECTS' INTERACTION TECHNIQUE PREFERENCE

1 - least preferred, 4 - most preferred

\begin{tabular}{llllllllllllllll}
\hline Test subj. & 1 & 2 & 3 & 4 & 5 & 6 & 7 & 8 & 9 & 10 & 11 & 12 & 13 & 14 & Total \\
\hline Stylus & 2 & 4 & 2 & 2 & 3 & 4 & 4 & 4 & 2 & 4 & 4 & 3 & 2 & 2 & 42 \\
Finger & 4 & 3 & 4 & 3 & 4 & 3 & 2 & 2 & 3 & 3 & 3 & 4 & 1 & 3 & 42 \\
Button & 3 & 2 & 3 & 4 & 2 & 2 & 1 & 3 & 4 & 1 & 1 & 1 & 3 & 4 & 34 \\
Paper & 1 & 1 & 1 & 1 & 1 & 1 & 3 & 1 & 1 & 2 & 2 & 2 & 4 & 1 & 22 \\
\hline
\end{tabular}

TABLE II. MANN-WiTHNEY TEST

Resulting p-values $(\mathrm{CF}=95 \%)$, significant values in italics.

\begin{tabular}{llll}
\hline Int. tech. & Paper & Buttons & Finger \\
\hline Stylus & 0,0012 & 0,2064 & 0,9817 \\
Finger & 0,0016 & 0,2064 & - \\
Buttons & 0,0565 & - & - \\
\hline
\end{tabular}


information compared to paper. This made it hard to get a good overview, especially for heavily medicated patients with a complex medical history.

\section{1) Stylus}

a) Observations: The usage of the stylus interaction technique mostly revealed ergonomic usability problems. A few physicians had problems locating the stylus in the PDA. Others had problems taking it out of the PDA and some physicians fiddled with the stylus during the patient conversation. We also observed a few incidents where the stylus "got lost" in the lab coat pocket because the user placed the handheld device and the stylus in the pocket separately.

b) Interview: Some physicians experienced the stylus interface as faster and more accurate than finger and button. One physician pointed out that he was "not as distracted from the patient with this one as for the other two [finger and button]". However, many physicians considered the stylus as an extra thing to be dependent on, and to get distracted of. They were also worried about the stylus being lost or misplaced. As one of them said: "Physicians are very good at misplacing things and put things in their pockets, so the stylus will be lost the first day. Your finger will probably not." He also pointed out that they "will need a big box of styluses". Some also commented that it was awkward to get the stylus in and out of the PDA every time they used it.

c) Card ranking: Despite the problems, stylus was ranked as most preferred by the physicians. A total of 6 ranked it highest. None ranked it as the least preferred. However, there were only significant differences with paper.

\section{2) Finger}

a) Observations: In general the physicians managed to use the finger interaction technique without larger problems. However, we observed a few occasions of pressure and accuracy problems during the ward rounds with finger. In a few cases the physician were able to operate the device with only one hand. We did not see this for the other interaction techniques.

b) Interview: The main argument in favor of the finger interaction technique was that the physicians were "not dependent on a separate tool [the stylus] because you always have your finger with you". Many pointed out that the interaction with the PDA is not a problem as long as the screen and buttons are large enough. When comparing finger and button many users liked that they could directly select their target without clicking through several steps to reach it. However, for many users finger felt inaccurate and some were worried about how much pressure to apply to the touch screen. In addition some users felt that their fingertip was too large for some user interface elements. They felt that the buttons was hidden under the finger and that they had to do an extra check before they pressed them. One commented that "if [the buttons] gets very small, I'll have to use my nail instead of the finger". Another physician was worried about hygiene if the screen got dirty. c) Card ranking: Finger was ranked as the second most preferred interaction technique. Four physicians ranked this method highest and one ranked it lowest.

\section{3) Button}

a) Observations: The button interaction technique caused most problems. Common mistakes were that they navigated the marker too far, or that they accidentally double-tapped the button. Another common mistake was that they used the finger to press the on-screen buttons even if they were instructed to only use the hardware buttons. In general the users spent longer time to finish their tasks with this interaction technique. When using the other interaction techniques, the physicians often used their finger, stylus or analog pen as a reference point in the device or chart to keep track of their reading position in the information. With the button technique this was harder because their fingers were occupied with the hardware buttons.

b) Interviews: Some users commented that user interface "was like a mobile phone", a device they felt comfortable with. With this interaction technique they could use their finger, and even one hand. They liked the immediate physical and audible (click) feedback from the buttons, in addition to the marker indicating the current selection. Some experienced button as more secure, because they were forced to confirm their selection with a click on the center-key. With the other techniques they feared selections by accident.

A few physicians found button unsuitable for longer list, because it caused a lot of button presses. Several considered this interaction technique as more distractive. As one said it; "I noticed that I had to change my focus, gaze or thoughts from a screen to a keyboard", because they had to constantly move their focus from the screen to the device buttons and back again.

The colored marker was not enough feedback for some users; "Even if the color changes, you are not sure where you are". Another problem was that the physicians had to move the marker towards what they wanted. With the other interaction techniques they could go there directly.

c) Card ranking: Button was the least preferred of the PDA-based interaction methods. Yet, it was ranked better than paper. Three ranked it as their most preferred method and equally many ranked it as their least preferred.

\section{4) Paper}

a) Observation: The physicians showed a variety of holding techniques when standing and writing in the paper chart. Usually writing while standing did not seem to bother them too much. However, in some cases the users experienced problems when writing in the chart; they got tired in their arm and were forced to change their holding position several times, or they were distracted by their IDcard, which was placed around the neck in a line and obstructed their writing. Another source of minor distractions was the pen. Sometimes the physicians had to search for it, other times they had to click it to get it working. 
b) Interview: The paper chart was the interaction technique most users were confident with. They commented that it provided better overview because more information was available on the page, such as blood pressure, heart rate, and allergies. Flexible input with the "analog" pen, freedom to make notes, and the ability to easily share the paper with colleagues was appreciated.

On the other hand, many physicians commented that handwriting made it easier to make mistakes, harder to correct them, and more difficult to interpret the information. They also commented on the greater risk of medication errors and interactions, or that they had to take heavy reference books with them. As one physician said; " (...) its more that you don't get the same quality of information. It is hard to write medications, they have complex names. It could be nice to have some support". In addition, the information registered in the paper chart cannot be reused by other systems, so it has to be registered twice. As one physician said; "Quick and easy to use, but more work afterwards." They also had to remember things such as medication name and dosage, rather than selecting from a list.

Some younger physicians commented that the patient chart was large and good to hide behind and less disturbing than PDA. In addition they liked that there was no chance of crashes or software malfunctions; "the paper chart has its advantages; it works as long as you can write".

c) Card ranking: Paper was ranked significantly lower than the PDA based interaction techniques. Only one ranked it as most preferred while 9 ranked it as the least preferred.

\section{DISCUSSION}

The study provides both insight into the general attitude of physicians towards PDA-based clinical information systems, and their opinions and preferences concerning different input techniques. While the particular findings about each specific interaction technique can be of interest to usability practitioners when selecting the primary form of interaction with the system, we have acquired an

\section{TABLE III. FACTORS THAT INFLUENCED THE USERS' PREFERENCES}

Factors for each interaction technique. For each factor, a plus indicate that the interaction technique had positive qualities, a minus indicate negative qualities (based on observations, physician interviews and patient focus group).

\begin{tabular}{lcccc}
\hline Factors influencing the users' preference & Stylus & Finger & Button & Paper \\
\hline Reliability (software malfunctions) & - & - & - & + \\
Information readability and overview & - & - & - & + \\
Patient experience & - & - & - & + \\
Flexible usage & - & - & - & + \\
Error prevention and decision support & + & + & + & - \\
Need for additional tool (pen/stylus) & - & + & + & - \\
Mapping between input/output & + & + & - & + \\
Physical size & + & + & + & - \\
Accuracy & + & - & + & + \\
One handed usage & - & + & - & - \\
Efficiency & + & + & - & + \\
Physical feedback & - & - & + & + \\
Feeling of security & - & - & + & + \\
Redundant registration & + & + & + & - \\
\hline
\end{tabular}

understanding of the aspects of importance for physicians when selecting and accepting handheld point-of-care systems. These factors, and the way each interaction technique satisfies them, are summed up in Table III.

Looking at the general assessment of physicians' attitude towards information devices in clinical practice, the study shows, that while some reservations and fear for potential problems exists, today's physicians are open for future implementations of PDA-based systems. While physician hold strong expectations about the advantages of such systems, e.g. to facilitate information lookup, it is important to scrutinize and consider the raised factors, e.g. patient experience and software malfunction, when designing a system for routine usage.

The comparison of the different input techniques reveals that physicians do have strong opinions about the input techniques they use and that these opinions vary widely between individuals. This is very clearly demonstrated by the fact that even the least preferred input technique is still most preferred by more than one fifth of the participants. Given the very simple usage pattern in the tests, it is even imaginable, that user preference ranking might be reversed for different usage patterns, e.g. scrolling long lists or multi-selection. Moreover, new technologies, such as touch screens with better precision and tactile feedback [24], together with increased popularity, will make the views on mobile devices and appropriate interaction techniques evolve quickly.

Some of the emerged issues with the different input techniques could be attributed to lacking experience with PDAs and the medication system and could be expected to vanish with increasing usage experience. But we think it is of major importance to realize the significant role of input techniques when designing any mobile information system for point-of-care usage and to include the selection of input techniques as an essential part of the interaction design process. Given the large variation in users' preferences it often seems advisable to provide the system with multiple input techniques and leave the final choice to the user to accommodate their individual needs. However, this requires the user interface to be tailored to accommodate all the interaction techniques, which may require suboptimal and costly solutions.

\section{A. Methodological Limitations}

One validity constriction of the study is that the patients were not real patients - they were carefully instructed actors and were not sufferers of the medical problem they simulated. However, when asking the physicians about the realism of the simulations they all gave a positive response. In addition, judging from the observations, most of them seemed to be "on duty" and asking questions and acting like physicians do. On the other hand the patient actors reported that they might have acted differently if they were real patients.

Another validity issue is that the medication system used in the simulated ward rounds did not compare to reality. The functionality was too sparse, and the user interface too simplistic. Interestingly, only a very few physicians commented on this - the simple system offered exactly what 
they needed. The reason was that the scenarios and patient cases were carefully aligned to the functionality offered in the prototypes.

It also has to be noted, that due to the recruitment process the participants' willingness to use new information systems probably was above average of the whole professional group.

\section{CONCLUSIONS}

In this comparative evaluation study we have used simulated ward rounds with physicians using a paper version and three PDA versions of a medication prescription system using stylus, finger and button interaction.

The great variance between the different interaction techniques makes it hard to select which technique a PDAbased point-of-care medication system should employ. Since technology is evolving and user preferences are bound to shift, the important contribution in this study is the factors that affects physicians' (and patients') acceptance of a mobile point-of-care system.

The results show that PDA-based systems have some qualities that make physicians willing to replace their paper based medication systems. Paper had a number of benefits compared to PDA; the users were confident using it, it provided better information overview, and it supported the patient-physician dialogue better. However, no undo mechanism and poor error prevention on paper made the physicians prefer the PDA-based solutions, despite generally low experience with handhelds and sparse training on the information system.

This study can inform the design and choice of interaction techniques on new handheld point-of-care systems.

\section{REFERENCES}

[1] Fischer, S., Stewart, T.E., Mehta, S., Wax, R., Lapinsky, S.E.: Handheld Computing in Medicine. JAMIA 10 (2003) 139

[2] Garritty, C., Emam, K.E.: Who's Using PDAs? Estimates of PDA Use by Health Care Providers: A Systematic Review of Surveys. Journal of Medical Internet Research 8 (2006)

[3] Lu, Y.C., Xiao, Y., Sears, A., Jacko, J.A.: A review and a framework of handheld computer adoption in healthcare. IJMl I74 (2005) 409-422

[4] Ash, J.S., Berg, M., Coiera, E.: Some unintended consequences of information technology in health care: the nature of patient care information system-related errors. Journal of the American Medical Informatics Association 11 (2004) 104-112

[5] Wagner, I.: A web of fuzzy problems: confronting the ethical issues. Commun. ACM 36 (1993) 94-101
[6] Bardram J.E., Bossen, C.: Mobility Work: The Spatial Dimension of Collaboration at a Hospital. CSCW 14 (2005) 131-160

[7] Shneiderman, B., Plaisant, C.: Designing the User Interface: Strategies for Effective Human-Computer Interaction. Addison Wesley (2004)

[8] Nielsen, J.: Usability Engineering. Morgan Kaufmann, 1993

[9] Bewley, W.L., Roberts, T.L., Schroit, D., Verplank, W.L.: Human factors testing in the design of Xerox's 8010 "Star" office workstation. Hum. Fact. in Comp. Sys. (1983) 72-77

[10] Svanæs, D., Alsos, O.A., Dahl, Y.: Usability testing of mobile ICT for clinical settings: Methodological and practical challenges. Int J Med Inform (2008)

[11] Alsos, O. A., Dabelow, B., Faxvaag, A.: "Doctors' Concerns of PDAs in the Ward Round Situation." Methods of Information in Medicine, 48(6), 2009

[12] Heath, C., Nicholls, K., Body movement and speech in medical interaction, Cambridge University Press Cambridge, 1986.

[13] Heath, C., Svensson, M. S., Hindmarsh, J., Luff, P. and Vom Lehn, D. Configuring awareness. Computer Supported Cooperative Work (CSCW), 11, 3 2002, 317-347.

[14] Margalit, R. S., Roter, D., Dunevant, M. A., Larson, S., Reis, S. "Electronic medical record use and physicianñpatient communication: An observational study of Israeli primary care encounters." Patient Education and Counseling 61(1): 134-141. (2006)

[15] Silvey, G.M., Macri, J.M., Lee, P.P., Lobach, D.F.: Direct Comparison of a Tablet Computer and a Personal Digital Assistant for Point-ofCare Documentation in Eye Care. AMIA Annual Symposium Proceedings, (2005) 689

[16] Alsos, O.A.: Exploring interface metaphors for using handhelds and PCs together, Master thesis, NTNU (2005)

[17] Lane, S.J., Heddle, N.M., Arnold, E., Walker, I.: A review of randomized controlled trials comparing the effectiveness of hand held computers with paper methods for data collection. BMC Medical Informatics and Decision Making 6 (2006) 23

[18] Dahl, Y., Svanaes, D., Nytro, O.: Designing Pervasive Computing for Hospitals: Learning from the Media Affordances of Paper-Based Medication Charts. Pervasive Health Conference and Workshops, (2006) $1-10$

[19] Pascoe, J., Ryan, N., Morse, D.: Using while moving: HCI issues in fieldwork environments. ACM TOCHI 7 (2000) 417-437

[20] Melby, L.: Prat, penn og papir: En sosiologisk analyse av medisinsk informasjonsbruk i en mobil praksis. PhD thesis, NTNU (2007)

[21] Kjeldskov, J., Skov, M.B., Als, B.S., Hoegh, R.T.: Is It Worth the Hassle? Exploring the Added Value of Evaluating the Usability of Context-Aware Mobile Systems in the Field. LNCS (2004) 61-73

[22] Alsos, O.A., Dahl, Y.: Toward a best practice for laboratory-based usability evaluations of mobile ICT for hospitals. NordiCHI 2008, ACM New York, USA (2008) 3-12

[23] Glaser, B.G., Strauss, A., The Discovery of Grounded Theory: Strategies for Qualitative Research. Aldine Transaction; 1967.

[24] Hoggan, E., Brewster, S.A., Johnston, J., Investigating the effectiveness of tactile for mobile touchscreens, Proceeding of $\mathrm{CHI}$ 2008 